\title{
Persistent current of one-dimensional perfect rings under the canonical ensemble
}

\author{
Man-Kit Yip, Jiu-Ren Zheng, ${ }^{*}$ and Ho-Fai Cheung \\ Department of Physics and Materials Science, City University of Hong Kong, Hong Kong
}

(Received 12 April 1995; revised manuscript received 18 August 1995)

\begin{abstract}
We have analyzed the harmonic contents of the persistent current at high temperatures under the canonical ensemble. Results show that the behavior of each harmonic is different from that under the grand-canonical ensemble. The persistent current of the multiring system is also presented.
\end{abstract}

\section{INTRODUCTION}

Modern studies on persistent current in mesoscopic rings have been renewed by Büttiker, Imry, and Landauer ${ }^{1}$ in 1983. More detailed quantitative calculations have been given by Cheung et al. ${ }^{2-4}$ Experimental measurements have been reported by various authors. ${ }^{5-7}$ One of the main discoveries not predicted by former theories at that time was that for an ensemble of $10^{7}$ three-dimensional (3D) rings the persistent current varies with magnetic flux with a dominant period of $h / 2 e$ ( $h c / 2 e$ in Gaussian units). This contrasts to that for a single ring whose dominant period is $h / e\left(=\Phi_{0}\right.$, the magnetic flux quantum). This was subsequently explained $^{8-12}$ by assuming that the number of electrons in each ring is fixed and independent of flux. That is, canonical ensemble should be used if the average properties of these rings are to be calculated.

The variation of the persistent current in one- and higherdimensional perfect and disorder rings as a function of temperature has been studied by Cheung et al. ${ }^{2-4}$ All these studies adopted the grand-canonical ensemble, so the chemical potential is fixed and the electron occupation probability follows the Fermi-Dirac distribution. When $T=0$ with the number of electrons fixed, studies have been carried out by Bouchiat and Montambaux, ${ }^{8}$ von Oppen and Riedel, ${ }^{9}$ Schmid, ${ }^{10}$ and Altshuler, Gefen, and Imry. ${ }^{11}$ Their calculation logic is to allow the chemical potential to vary with magnetic flux such that the number of electrons remains fixed. This procedure is perfectly correct at $T=0$. At $T \neq 0$, the persistent current under the exact canonical ensemble has not been calculated so far. The reason is that the canonical-ensemble occupation probability is complicated and sensitive to the details of the energy levels. Most of the time the canonical-ensemble occupation probability is not known.

At high temperatures, if one approximates the canonicalensemble occupation probability by the Fermi-Dirac distribution with a suitably chosen chemical potential, one might expect to get a reasonable answer for the persistent current. The reason might be that in the high-temperature limit, canonical ensemble and grand-canonical ensemble give the same occupation probability. In this paper we present exact analysis on the persistent current on 1D perfect rings under the canonical ensemble. Our results show that replacing the canonical-ensemble occupation probability with the FermiDirac distribution cannot lead to correct results for the persistent current. Our results send warnings to those using the above approximation.

\section{THE MODEL}

The model we consider is $1 \mathrm{D}$ perfect rings with noninteracting electrons threaded by a magnetic flux. The wave vector and energy of the eigenfunctions are given by

$$
\begin{gathered}
k_{n}=\frac{2 \pi}{L}\left(n+\frac{\Phi}{\Phi_{0}}\right), \\
E_{n}=\frac{\hbar^{2}}{2 m}\left[\frac{2 \pi}{L}\left(n+\frac{\Phi}{\Phi_{0}}\right)\right]^{2},
\end{gathered}
$$

where $\Phi$ is the magnetic flux through the loop. The corresponding current is given by

$$
I_{n}=-\frac{e \hbar}{m L}\left[\frac{2 \pi}{L}\left(n+\frac{\Phi}{\Phi_{0}}\right)\right]
$$

where $n=0, \pm 1, \pm 2, \ldots$ The energy versus wave-vector relation is described by a parabola. As the flux increases, levels on the left-hand side move down the parabola, move across to the right-hand side, and then move up the parabola on the right-hand side. If there are many electrons in the system, the $E$ versus $k$ parabolic relation near the Fermi energy can be approximated by two linear branches. Let us rewrite the energy levels on the left branch and right branch as

$$
\begin{aligned}
& E_{\text {left }}=\Delta\left(n-\frac{\Phi}{\Phi_{0}}\right), \\
& E_{\text {right }}=\Delta\left(n+\frac{\Phi}{\Phi_{0}}\right),
\end{aligned}
$$

where $n$ can take $-\infty$ to $+\infty$ and $\Delta$ is the energy spacing on either branch. The current of each level on the left branch and right branch is $+I_{0}$ and $-I_{0}$, respectively. Electron levels have twofold spin degeneracy. In our analysis we consider $g$-fold degenerate levels.

We noticed that the above model is equivalent to a model of small metallic particles studied by Denton, Mühlschleger, and Scalapino. ${ }^{13}$ If the energy levels are uniformly spaced and a magnetic field is applied, the effect of the magnetic 
field is to shift the spin-up levels downward and the spindown levels upward linearly. So the energies of the spin-up and spin-down levels can be written as

$$
\begin{gathered}
E_{\text {up }}=n \Delta-h, \\
E_{\text {down }}=n \Delta+h,
\end{gathered}
$$

where $n$ takes the value $-\infty$ to $+\infty . \Delta$ is the energy spacing for spin-up levels (or spin-down levels), $h$ is the external magnetic field measured in suitable energy units. The magnetic moment $M$ of each level is +1 and -1 (measured in suitable units) for spin-up and spin-down states, respectively. The energy levels of the two models are equivalent. Furthermore, the magnetic moment of all the electrons in the metallic-particle model is almost equivalent to the persistent current in the 1D perfect-ring model. They are not exactly equivalent because in the metallic-particle model the spin-up energy levels are not linked to the spin-down energy levels, whereas in the 1D perfect-ring model the left branch is actually connected to the right branch at the very bottom of the branches. The correct relation between them is

$$
\frac{\langle I\rangle}{I_{0}}=\langle M\rangle-\frac{2 g h}{\Delta}
$$

When using this equation to find the persistent current for 1D perfect rings, all the parameters in the metallic-particle model should be substituted by the corresponding parameters in the perfect-ring model (i.e., $h \rightarrow \Delta \Phi / \Phi_{0}$ ).

In the following, we first work out the partition function for the metallic-particle model under the canonical ensemble. From that we can calculate the average magnetic moment and subsequently we can deduce the persistent current. At low temperatures the persistent currents from canonical and grand-canonical ensembles are the same up to exponentially small corrections. This limit will not be discussed any further in this paper. Instead our objective is to investigate the hightemperature limit. Since the persistent current is periodic in the magnetic flux with period $\Phi_{0}$, we express the persistent current as a Fourier series. We work out the Fourier coefficients of the persistent current in the high-temperature limit.

Consider the metallic-particle model with energy levels given by Eq. (4), where $n$ goes from $-\infty$ to $\infty$. Let $\beta$ denote $1 / k_{B} T$, where $k_{B}$ is the Boltzmann constant and $T$ is the temperature. Following the calculation by Denton, Mühlschleger, and Scalapino, ${ }^{13}$ which is also described in an earlier paper by Chen and Cheung, ${ }^{14}$ the partition function can be written as

$$
Z(h)=\frac{1}{2 \pi i} \oint \frac{d z}{z} z^{r} \prod_{n=1}^{\infty}\left[1+z e^{-n \beta \Delta-\beta h}\right]^{g}\left[1+z e^{-n \beta \Delta+\beta h}\right]^{g} \prod_{n^{\prime}=0}^{\infty}\left(1+\frac{1}{z} e^{-n^{\prime} \beta \Delta-\beta h}\right)^{g}\left(1+\frac{1}{z} e^{-n^{\prime} \beta \Delta+\beta h}\right)^{g},
$$

where $r$ is the number of holes of the ground-state uppermost filled level. The value of $r$ is determined by the actual number of electrons in the system. Without loss of generality we take $r$ to be from 0 to $(2 g-1)$. Higher or lower value of $r$ can be deduced because the energy-level ladders are translational invariant. Following the procedures and the mathematical identity mentioned in Denton, Mühlschleger, and Scalapino, ${ }^{13,15}$ one obtains

$$
Z(h)=\frac{1}{2 \pi} Z_{B}^{2 g} q^{-g / 2} \int_{-\pi}^{\pi} d \phi e^{i(r-g) \phi}\left(\sum_{n^{\prime}=-\infty}^{\infty} q^{(n+1 / 2)^{2}} e^{i(n+1 / 2)(\phi+i \beta h)}\right)^{g}\left(\sum_{n=-\infty}^{\infty} q^{(n+1 / 2)^{2}} e^{i(n+1 / 2)(\phi-i \beta h)}\right)^{g},
$$

where $q=e^{-\beta \Delta / 2}$ and $Z_{B}=\prod_{m=1}^{\infty}\left(1-q^{2 m}\right)^{-1}$. We calculate the sum over $n$ by using the Poisson summation formula, then $Z(h)$ can be written as

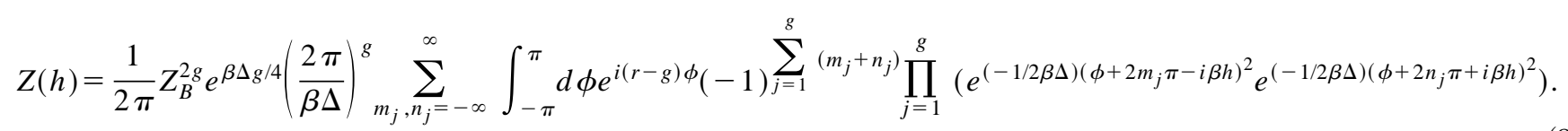

We put in the condition $-g \leqslant \Sigma\left(m_{j}+n_{j}\right)<g$ so that the integration limits could be extended from $-\infty$ to $\infty$. After completing the square with respect to $\phi$ in the exponent, the integration can be carried out readily. The result is

$$
\begin{aligned}
Z(h)= & \frac{1}{\sqrt{2 g}}\left(\frac{2 \pi}{\beta \Delta}\right)^{g-1 / 2} Z_{B}^{2 g} e^{\beta \Delta r / 2-\beta \Delta r^{2} / 4 g+g \beta h^{2} / \Delta} \sum_{-g \leqslant \Sigma_{j=1}^{g}\left(m_{j}+n_{j}\right)<g} \exp \left(\frac{-2 \pi^{2}}{\beta \Delta} \sum_{j=1}^{g}\left(m_{j}^{2}+n_{j}^{2}\right)\right) \\
& \left.\times \exp \left[\frac{\pi^{2}}{g \beta \Delta}\left(\sum_{j=1}^{g}\left(m_{j}+n_{j}\right)\right)^{2}\right] \exp \frac{i 2 \pi h}{\Delta} \sum_{j=1}^{g}\left(m_{j}-n_{j}\right)\right) \exp \left(\frac{-i r \pi}{g} \sum_{j=1}^{g}\left(m_{j}+n_{j}\right)\right)
\end{aligned}
$$

The second last term determines the order of the harmonics. The $l$ th harmonic comes from the term where $\Sigma\left(m_{j}-n_{j}\right)= \pm l$. After comparing their magnitudes and considering all possible combinations, the leading term for each harmonic in the high-temperature limit can be sorted out. In this limit $Z(h)$ can be expressed as

$$
Z(h)=\frac{1}{\sqrt{2 g}} Z_{B}^{2 g}\left(\frac{2 \pi}{\beta \Delta}\right)^{g-1 / 2} e^{\beta \Delta r / 2-\beta \Delta r^{2} / 4 g+g \beta h^{2} / \Delta}\left(1+\sum_{l=1}^{\infty} \frac{4 C_{k}^{g}}{1+\delta_{k, g}} e^{\left(-\pi^{2} / \beta \Delta g\right)\left[l^{2}+2 k(g-k)\right]} \cos \frac{\pi r l}{g} \cos \frac{2 \pi l h}{\Delta}\right)
$$


where $p$ and $k$ are defined by $l=p g+k$ with $p=0,1,2, \ldots$. and $k=1,2, \ldots, g$. This is the final expression for the canonical-ensemble partition function. Next we deduce the average magnetic moment and then the persistent current of the $1 \mathrm{D}$ perfect-ring model.

\section{PERSISTENT CURRENT IN THE RING}

It is possible to calculate the average magnetic moment from the occupation probability of the levels. A more direct approach is to find the magnetic Gibbs function $G^{*}=-(1 / \beta) \ln Z(h)$, then the average magnetic moment (or magnetization up to some volume) can be found from the relation $\langle M\rangle=-\left(\partial G^{*} / \partial h\right)_{T}$. After expanding the logarithmic function, taking the derivative, and then considering all the combinations carefully, we finally obtained the average magnetic moment as

$$
\begin{aligned}
\langle M\rangle= & \frac{2 g h}{\Delta}+\sum_{l=1}^{\infty} \frac{(-1)^{p+1}}{p \delta_{k, g}+1} \frac{8 \pi l}{\beta \Delta} \frac{C_{k}^{g}}{1+\delta_{k, g}} \\
& \times e^{\left(-\pi^{2} / \beta \Delta g\right)[l g+k(g-k)]} \cos \frac{\pi r l}{g} \sin \frac{2 \pi l h}{\Delta} .
\end{aligned}
$$

As expected, the dominant term in the average magnetic moment is linear in magnetic field. Using the mapping between the metallic-particle model and the 1D perfect-ring model [i.e., Eq. (5)], the persistent current of $1 \mathrm{D}$ perfect rings is

$$
\begin{aligned}
\frac{\langle I\rangle}{g I_{0}}= & \sum_{l=1}^{\infty} \frac{(-1)^{p+1}}{p \delta_{k, g}+1} \frac{8 \pi l}{\beta \Delta g} \frac{C_{k}^{g}}{1+\delta_{k, g}} e^{\left(-\pi^{2} / \beta \Delta g\right)[l g+k(g-k)]} \\
& \times \cos \frac{\pi r l}{g} \sin \frac{2 \pi l \Phi}{\Phi_{0}}
\end{aligned}
$$

The current is expressed as a Fourier series, showing all the harmonics. Only the sine terms exist, reflecting the symmetry of the persistent current when the magnetic flux is reversed. For the same model using the grand-canonical ensemble (keeping the same average number of electrons) would lead to the following result: ${ }^{2}$

$$
\frac{\langle I\rangle}{g I_{0}}=\frac{8 \pi}{\beta \Delta} \sum_{l=1}^{\infty} e^{-2 l \pi^{2} / \beta \Delta} \cos \left(l k_{F} L\right) \sin \left(\frac{2 l \pi \Phi}{\Phi_{0}}\right) .
$$

In both cases every harmonic decreases exponentially with temperature. The dominant term is the $l=1$ term. We note that this term is proportional to $\exp \left[-(2 g-1) \pi^{2} / \beta \Delta g\right]$ in the canonical ensemble, but is proportional to $\exp \left(-2 \pi^{2} / \beta \Delta\right)$ in the grand-canonical ensemble. The current amplitudes are very different even though the occupation probabilities of the levels at high temperatures approach each other under the two ensembles. The two exponential factors agree with each other only in the high-degeneracy limit. We also noted that the amplitude of the harmonics is roughly $\exp \left(-2 l \pi^{2} / \beta \Delta\right)$ in the grand-canonical ensemble. The ratios of these exponential factors are the same for all pairs of adjacent harmonics, whereas in the canonical ensemble the amplitude of the harmonics is roughly exp $\left\{-\pi^{2}[\lg +k(g-k)] / \beta \Delta g\right\}$, and so the ratios of these exponential factors are not the same for different pairs of adjacent harmonics.

In experiments that measured persistent current, ${ }^{5-7}$ a constant magnetic flux and a small time-varying flux are applied. In this way the amplitude for the first few harmonics can be deduced. The spin-degeneracy is usually lifted by the applied magnetic field. But if the magnetic field does not act on the ring (only through the empty space enclosed by the ring) then the levels would still have a spin degeneracy of 2. For single-ring systems, our results show that if $g=2$, the amplitude of the first two harmonics ( $l=1$ and 2 ) should be proportional to $\exp \left(-3 \pi^{2} / 2 \beta \Delta\right)$ and $\exp \left(-2 \pi^{2} / \beta \Delta\right)$ in the canonical ensemble, $\exp \left(-2 \pi^{2} / \beta \Delta\right)$ and $\exp \left(-4 \pi^{2} / \beta \Delta\right)$ in the grand-canonical ensemble. If the amplitudes for the first two harmonics are measured as a function of temperature, it would be possible to deduce whether canonical or grand-canonical ensemble is more appropriate for the particular experimental condition. We believe this could be a sensitive test.

For systems with many rings, the electron number in each ring may not be the same. One has an ensemble of rings with different $r$, where $r$ can vary from 0 to $2 g-1$. Taking an average over all possible values of $r$, most of the harmonics shown in Eq. (12) vanish. However, some higher-order terms which are not shown in Eq. (12) survive. We found that all the odd harmonics of the average current are zero. All the nonzero terms are described by $l=2 l^{\prime}$, where $l^{\prime}=1,2,3, \ldots$ Defining $p^{\prime}$ and $k^{\prime}$ by $l^{\prime}=p^{\prime} g+k^{\prime}$ where $p^{\prime}=0,1,2, \ldots$ and $k^{\prime}=1,2, \ldots, g$, the average persistent current of multiring systems is

$$
\begin{aligned}
\frac{\langle\langle I\rangle\rangle}{g I_{0}}= & \sum_{l^{\prime}=1}^{\infty} \frac{8 \pi l^{\prime}}{\beta \Delta g} \frac{\left(C_{k^{\prime}}^{g}\right)^{2}}{1+\delta_{k^{\prime}, g}} \frac{2 p^{\prime}+1}{\left(2 p^{\prime 2}+3 p^{\prime}\right) \delta_{k^{\prime}, g}+1} \\
& \times e^{\left(-2 \pi^{2} / \beta \Delta g\right)\left[g l^{\prime}+k^{\prime}\left(g-k^{\prime}\right)\right]} \sin \frac{4 l^{\prime} \pi \Phi}{\Phi_{0}} .
\end{aligned}
$$

Therefore the period would cross over from $\Phi_{0}$ for singlering systems to $\Phi_{0} / 2$ for multiring systems. This result agrees with previous analysis ${ }^{8-10}$ on $g=1$ perfect and disorder rings at zero temperature. Analysis starting from Eq. (9) shows that this crossover of period is true over the whole range of temperatures.

The realization of $1 \mathrm{D}$ perfect rings using semiconductors may not be that far away. It would be most interesting to have experiments on both single-ring and multiring systems with the same type of rings. Right now the calculation is on 1D perfect rings. Whether similar features occur at higherdimensional rings with disorders is still open for investigation.

\section{ACKNOWLEDGMENT}

This work was supported by the Hong Kong UGC Research Grants Council under Grant No. 904033. 
*Permanent address: Centre for Fundamental Physics, University of Science and Technology of China, Hefei, Anhui 230026, People's Republic of China.

${ }^{1}$ M. Büttiker, Y. Imry, and R. Landauer, Phys. Lett. 96A, 365 (1983).

${ }^{2}$ H. F. Cheung, Y. Gefen, E. K. Riedel, and W. H. Shih, Phys. Rev. B 37, 6050 (1988).

${ }^{3}$ H. F. Cheung, Y. Gefen, and E. K. Riedel, IBM J. Res. Dev. 32, 359 (1988).

${ }^{4}$ H. F. Cheung, E. K. Riedel, and Y. Gefen, Phys. Rev. Lett. 62, 587 (1989).

${ }^{5}$ L. P. Levy, G. Dolan, J. Dunsmuir, and H. Bouchiat, Phys. Rev. Lett. 64, 2074 (1990).

${ }^{6}$ V. Chandrasekar, R. A. Webb, M. J. Brady, M. B. Ketchen, W. J. Gallagher, and A. Kleinsaaer, Phys. Rev. Lett. 67, 3578 (1991).
${ }^{7}$ D. Mailly, C. Chapelier, and A. Benoit, Phys. Rev. Lett. 70, 2020 (1993).

${ }^{8}$ H. Bouchiat and G. Montambaux, J. Phys. (Paris) 50, 2695 (1989).

${ }^{9}$ F. von Oppen and E. K. Riedel, Phys. Rev. Lett. 66, 84 (1991).

${ }^{10}$ A. Schmid, Phys. Rev. Lett. 66, 80 (1991).

${ }^{11}$ B. L. Altshuler, Y. Gefen, and Y. Imry, Phys. Rev. Lett. 66, 88 (1991).

${ }^{12}$ Electron-electron interaction has also been proposed to explain this. See, for example, V. Ambegaokar and U. Eckern, Phys. Rev. Lett. 65, 381 (1990).

${ }^{13}$ R. Denton, B. Mühlschleger, and D. J. Scalapino, Phys. Rev. B 7, 3589 (1973).

${ }^{14}$ H. P. Chen and H. F. Cheung, J. Phys. C 7, 6707 (1995).

${ }^{15}$ E. T. Whittaker and G. N. Watson, A Course of Modern Analysis (Cambridge University Press, London, 1935). 